\title{
An Efficient Optimization based Vehicle Movement Prediction with Aid of Feed Forward Back Propagation Neural Network
}

\author{
E. Baby Anitha \\ Assist.Prof.,Department of CSE \\ K.S.R College of Engineering \\ Tiruchengode
}

\author{
K. Duraiswamy, Ph. D \\ Dean,Department of CSE \\ K.S. Rangasamy College of Technology \\ Tiruchengode
}

\begin{abstract}
Moving vehicle location prediction method mainly based on their spatial and temporal data .The moving objects has been developed as a specific research area of Geographic Information Systems (GIS). Most of the techniques have been used for performing the vehicle movement detection and prediction process. This type of work is a lack of analysis in predicting the moving vehicles location in current as well as in the future. Existing methods are using a Genetic Algorithm (GA) and Particle Swarm Optimization algorithm (PSO) for finding optimal paths in moving objects. Within the previous technique, there's no guarantee for fulfillment to finding a vehicle optimal path and also still now wants to improvement for choosing optimal path. To beat the disadvantage in the existing method, during this paper, to propose moving vehicle location prediction algorithm is an Artificial Bee Colony algorithm $(\mathrm{ABC})$ and Feed Forward Back Propagation Neural Network (FFBNN). During this proposed algorithm is used for compute vehicle optimal path and selected optimal paths are given to the FFBNN to accomplish the training process. The trained FFBNN is then used to find the vehicle moving from the current location. By combining $\mathrm{ABC}$ algorithm and FFBNN, the moving vehicle's location is predicted more efficiently. The outcomes of the FFBNN-ABC algorithm are compared with results of previous method, such as FFBNNGA, FFBNN-PSO. The evaluation result shows that the proposed technique more accurate than other algorithms.
\end{abstract}

Keywords: Moving Vehicle Location Prediction, Artificial Bee Colony Algorithm (ABC), Feed Forward Back Propagation Neural Network (FFBNN), Frequent Paths, Genetic Algorithm (GA), and Particle Swarm Optimization Algorithm (PSO).

\section{INTRODUCTION}

In recent years, data mining and knowledge discovery have become some of the popular fields of researches. Spatial data mining is one of the requirements of data mining process spatial data [1]. Spatial data mining is the technique of extracting implicit information, economical, social and scientific problems, spatial relations, or other patterns not explicitly stored in spatial databases [3]. Spatial data mining has deep roots in both traditional spatial analysis fields (such as spatial statistics, analytical cartography, and exploratory data analysis) and various data mining fields in statistics and computer science (such as clustering, classification, association rule mining, information visualization and visual analytics [2]. In spatial data mining, the prediction of moving object's location based on the time series is a significant task in several applications such as wireless based, location-aware devices and networks of sensors and traffic analysis. The need for analysis, modeling and processing of traffic data is of much concern as the amount of moving vehicles are increasing speedily every day. Likewise, the extraction of additional information about traffic conditions, optional routes and possible prediction of troublesome situations, such as traffic jams, becomes necessary [4].

The Mathematical study of spatial objects and its geometric properties which are preserved under deformations such as bending and stretching are used in investigating the topology of a spatial object. The study of the topological changes of spatial objects over time, i.e., of time dependent geometries called moving objects, is important in several applications such as Geographical Information Systems (GIS), Spatiotemporal databases, the processing of animation images in multimedia applications and the topology control of Wireless Sensor Networks (WSN) [8].

In Mobile and Spatiotemporal databases, monitoring of continuous spatial queries over moving objects is necessary in various applications such as Public Transportation, Logistics, Location Based Services and more. In many applications, the moving vehicles location prediction plays an important role. Many methods were developed to find the vehicle location, which are briefly discussed in the following section. In such works, there is a lack of analysis in predicting the vehicles location. The vehicle's location is predicted by finding the topological relationships among trajectories and locations and also the GPS points are determined by $30 \mathrm{~m}$ circular window from the image error range. The error range of the image is not static; it varies from image to image. So this technique is not applicable to all images. Moreover, there is no standardization available for selecting the suitable input image. If all the aforesaid drawbacks in the literary works are solved, then the moving vehicle's location prediction performance is improved with higher accuracy [5].

In this study, a moving vehicle location prediction algorithm is proposed to predict the vehicle's future location from the current location using FFBNN-ABC technique. After gathering the vehicle's frequent paths from all the vehicles movement in a particular period, among the frequent paths, the vehicles optimal paths are computed using the $\mathrm{ABC}$ algorithm. After that selected optimal path for each vehicle are given to FFBNN for the training process. To get more accurateness in the prediction of vehicle's future location from the current location. $\mathrm{ABC}$ is used in the training process. The rest of the paper is organized as follows: Section 2 reviews the related works with respect to proposed method. Section 3 discuss about the proposed technique. Section 4 
shows the experimental result of the proposed technique and section 5 concludes the paper.

\section{RELATED WORKS}

Liu and Schneider [5] have developed a model which determines the topological changes of a complex moving region through snapshots called observations. They have introduced a two phase strategy: the first phase partitions the observations into several evaluation units and uniquely maps a unit before the change in exactly one unit after the modification and the second phase interprets the topological change by integrating all basic topological changes from evaluation units.

Shaw and Gopalan [6] have derived an application of the modified priori algorithm in coordinating sets of trajectories to find the frequent trajectory coordinates. In order to decrease the unnecessary search time and space, some additional steps have been added in the algorithm to prune the coordinating sets created. They have also explained the fundamentals of data origination process and database structure to hold the coordinate data sets and the implementation of the algorithm with the object oriented programming language by an illustration. The technique can be applied to motivating game domains to find the recurrent trajectory of an object shot by a player which follows a trajectory path.

Diansheng and Mennis [2] have proposed a graph-based approach that treats trajectory data as a complex network. They have also developed a technique that establishes the topological associations among trajectories and locations and utilizes a spatially constrained graph partitioning method to discover natural regions defined by trajectories which are within the context of vehicle movements. Moreover, it has been found that the discovered hierarchical regions can effectively facilitate the understanding of trajectory patterns and discovered the trajectory clusters that the existing methods failed to detect.

Ivana Nizetic et al. [12] have discussed a conceptual model for predicting moving object's future locations can be very useful in many application areas and data model of moving objects considering various object's characteristics. The location of the vehicle equipped with a GPS device can be received almost continually. The proposed approach has a great issue to predict moving object's next position.

Ajaya Kumar Akasapu et al. [8] have proposed a model for analyzing the trajectories of moving vehicles and develop the algorithm for mining the frequency patterns of Trajectory data .The trajectory data are normally obtained from locationaware devices that capture the position of an object at a specific time interval. The moving vehicle location prediction plays an important task in many applications. Different methods are developed to find the vehicle location. In our previous work we proposed GA and PSO optimization techniques for finding a vehicle optimal path. Both techniques are not a guarantee of success in finding an optimal path [13].Therefore, this method still now needs improvement for selecting an optimal path. The above mentioned literary works are resolvable, after that the moving vehicle's location prediction performance is improved with higher accuracy [16] [17].

\section{PROPOSED METHODOLOGY}

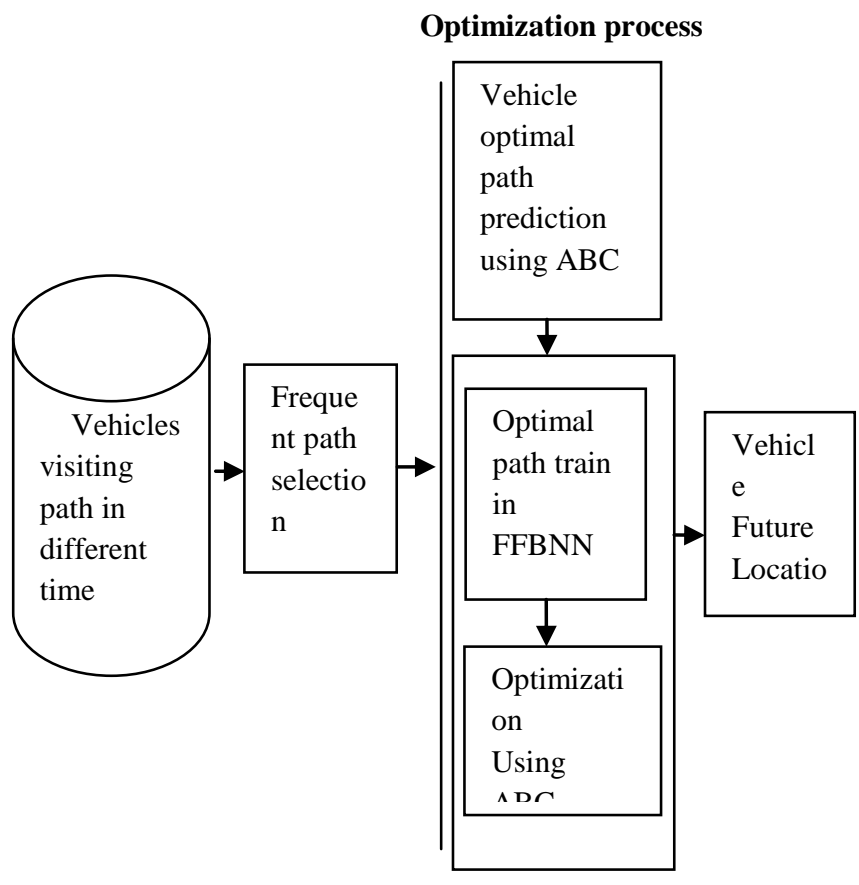

Fig1. Architecture of proposed prediction of moving Vehicle location.

The architecture of proposed prediction of moving vehicle location is shown in Fig 1. At the outset, the vehicles visiting paths in different time periods are collected and then compute frequent paths for each vehicle. After the frequent path are given as the input to the optimization process to select optimal paths for each vehicle. The most frequent paths are given as the input to FFBNN. While training, the FFBNN parameters are optimized using $\mathrm{ABC}$ algorithm. In testing process, more number of optimal paths is given to well train FFBNN-ABC to validate whether the given testing data predict the vehicles future location perfectly or not. The proposed methods contain the following phases is

\section{Frequent path selection, \\ 2. Optimization process.}

\subsection{Frequent Path Selection}

In this proposed method, the graph $G$ is intended with $n$ number of nodes (junctions) and the nodes are connected by the paths. The number of vehicles in the Designed graph is represented as $\mathrm{V}_{\mathrm{h}}=\left\{\mathrm{v} 1, \mathrm{v} 2 \ldots \mathrm{v}_{\mathrm{e}}\right\}$, where, e is a number of vehicles and the paths are represented as $P_{s}=\left\{p 1, p 2 \ldots p_{z}\right\}$. Moreover, the weights $\mathrm{Wn}$ for nodes are allocated within the arbitrary integer values $[1, \mathrm{w}]$. In a different time period $\mathrm{T}_{\mathrm{m}}=$ $\left\{\mathrm{T} 1, \mathrm{~T} 2 \ldots \mathrm{T}_{\mathrm{i}}\right\}$, the vehicles $\mathrm{v}_{\mathrm{e}}$ visited paths need to be collected. The designed graph is illustrated in Fig. 2.

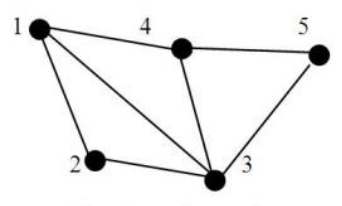

Fig. 2 sample graph
Node $\mathrm{n}=(\mathrm{n} 1, \mathrm{n} 2, \mathrm{n} 3, \mathrm{n} 4, \mathrm{n} 5)$

Vehicles $\mathrm{V}=(\mathrm{v} 1, \mathrm{v} 2, \mathrm{v} 3, \mathrm{v} 4, \mathrm{v} 5)$

Number of available Paths $P=7$

The vehicle $\mathrm{v} 1$ initially start traversing at the node 1 in a time period $\mathrm{T} 1$ and its next visit to the node 4 by utilizing the path $1->4$ and subsequently it visit the nodes 2 and 3 via the paths $1-$ 
$>2$ and 2->3respectively. In this same time period T1, the other vehicle's traversing paths are also to be computed. The same procedure is repeated for all vehicles in dissimilar time periods and the collected paths from the above mentioned graph in three time periods are listed in the Table 1.

\section{Table 1}

Different paths obtained in three time periods

\begin{tabular}{|c|c|l|}
\hline $\begin{array}{c}\text { Time } \\
\text { periods }\end{array}$ & \multicolumn{1}{|c|}{ vehicles } & \multicolumn{1}{|c|}{ Visited paths } \\
\hline T1 & V1,V2,V3,V4,V5 & $\begin{array}{l}(1,2,3),(4,5,3,2,1), \\
(2,3,4,1),(1,4,5), \\
(3,4,5)\end{array}$ \\
\hline T2 & V1,V2,V3,V4,V5 & $\begin{array}{l}(5,4,1,3),(1,3,5,4), \\
(3,4,1,2),(4,3,1), \\
(2,1,4)\end{array}$ \\
\hline & & $\begin{array}{l}(1,2,3,5,4),(2,3,4), \\
(5,3,2,1),(3,2,1,4,5), \\
(4,5,3,2)\end{array}$ \\
\hline
\end{tabular}

The vehicles are exploiting different paths to achieve the same target node in different time periods. Similarly, in different time periods, the vehicles $\mathrm{v}_{\mathrm{e}}$ visited paths are collected and the frequency value of path visited more than once by the particular vehicle $\mathrm{v}_{\mathrm{e}}$ is calculated. Based on the frequency value, the vehicle $v_{e}$ frequently visited paths $p$ is computed and an index value is allocated for each computed frequent path. The number of frequent paths for the vehicle $\mathrm{v}_{\mathrm{e}}$ is denoted as $\mathrm{fP}_{\mathrm{e}}^{\mathrm{v}}=\left\{\mathrm{p} 1, \mathrm{p} 2 \ldots \mathrm{p}_{\mathrm{j}}\right\}$ and the corresponding index value for the frequent paths is represented as $I_{e}^{v}=\{i 1$, i2 ...ix \}, where ix is a value based on the number of selected frequent paths for the vehicle $\mathrm{v}_{\mathrm{e}}$.

\subsection{Vehicle Optimal Path Prediction using $\mathrm{ABC}$}

To obtain the exact future location of the vehicles, first we select the optimal path from number of frequent path $\mathrm{fp}$. The optimal path selection by $\mathrm{ABC}$ algorithm finds the precise paths for all vehicles utilized to identify the vehicle future location.

The main steps of the algorithms are given as follows.

Step1. Initial food sources are produced for all employed bees

Step2. While stop criteria is not satisfied, go to step 3 to step

Step3. Send the employed bees onto their food sources.

Step4. Send the onlooker bees onto the food sources depending on their nectar amounts.

Step5. Send the scout bees to search possible new food sources.

Step6. Memorize the best food source found so far. Until (termination criterion are met)

$\mathrm{ABC}$ algorithm is a swarm based meta-heuristic algorithm that is inspired by the intelligent behavior of the honey bee foraging, this optimization algorithm was introduced by dervis karabega in 2005, and is based on inspecting the behaviors of real bees on finding nectar amounts and sharing the information of food sources to the other bees in hive. These specialized bees try to maximize the nectar amount stored in the hive by performing efficient division of labor and self organization [9]. It is a very simple, robust and population based stochastic optimization algorithm. The performance of $\mathrm{ABC}$ algorithm is compared with other algorithms such as GA and PSO [10].It consist of three components namely, employed bees, onlookers bees, scout bees. The employed bees are coupled with the food sources in the region of the hive and they transfer the data to the onlookers about the nectar quality of the food sources exploiting. Onlooker bees are looking the dance of the employed bees inside the hive to pick one food source to exploit according to the data provided by the employed bees [11]. The employed bees of an abandoned food source become a scout and seeking a new food source randomly. The number of food source denotes the location of portable solutions of the optimization problem and the nectar amount of a source denotes the quality of the solution [12].

Steps of the $\mathrm{ABC}$ algorithm are,

1. Randomly generate a set of solutions as initial food sources $Z_{i}, \mathrm{i}=(1,2 \ldots \mathrm{N})$. Set $\mathrm{E}_{\mathrm{S}}=U_{I=1}^{N}\left\{Z_{i}\right\}$ Where $\mathrm{E}_{\mathrm{s}}$ is the set of food sources and assigns each employed bee to a food source. Assign each employed bee to a food source.

2. Evaluate the fitness $f\left(Z_{i}\right)$ of each of the food sources.

3. Set cycle to 1

4. Repeat

5. For each employed bee

i) Produce new solution (using neighborhood operator) $\mathrm{v}_{\mathrm{i}}$ by using (6)

ii) Calculate the value $f\left(Z_{i}\right)$.

iii) Apply greed selection process.

6. Calculate the probability values $\mathrm{P}_{\mathrm{i}}$ for the solution $\left(\mathrm{Z}_{\mathrm{i}}\right)$ by using (5)

7. For each onlooker bee

i) Select a food source (solution) $\mathrm{Z}_{\mathrm{i}}$ depending on $\mathrm{Pi}$

ii) Produce new solution $v_{i}$

iii) Calculate the value of $f_{i}$

iv) Apply greedy selection process

8. If any abandoned solution is present, then replace a new solution, which will be randomly produced by (7).

9. Memorize the best solution so far.

10. Cycle $=$ cycle +1

11. Until cycle $=\mathrm{MCN}$.

The $\mathrm{ABC}$ algorithms an iterative algorithm and it starts by generating random solution as a food source $Z_{i}(1,2 \ldots N)$. Where $\mathrm{N}$ denotes the size of the population or the total number of food source and assigning each employed bee to a food source. Each solution is a D-dimensional vector. So $\mathrm{j}$ represents a particular dimension of a particular solution $(j=1$, $2 \ldots$ D).This generation process is called as initialization process. To evaluate the best food source position, the fitness value of the generated food source is calculated using the equation.

$$
\begin{aligned}
& F^{v m}(j)=\max \left(\mathrm{f}^{\mathrm{ix}}\right) \\
& \mathrm{f}^{\mathrm{ix}}=\mathrm{v}\left(\mathrm{p}_{\mathrm{j}}\right)+\left(\mathrm{w}_{\mathrm{n}-1} * \mathrm{w}_{\mathrm{in}}\right)
\end{aligned}
$$


Where $F^{v m}(j)$ is the fitness function of the $\mathrm{j}^{\text {th }}$ parameter generated for the vehicle $\mathrm{v}_{\mathrm{e}}$ and $\mathrm{f}^{\mathrm{ix}}$ is the individual fitness value of the index ix. In equation $(2), v\left(p_{j}\right)$ is the frequency value of frequency path $p_{j}$, which is presented in the index ix and $w_{n-1} * w_{n}$ are the weight values of nodes and the path $p_{j}$ is presented between these two nodes. After finding the fitness value, the iteration is set to 1.Afterward the phase of employed bee is carried out [15]

Employed bee phase: In the employed bee phase, new population parameters are generated using an equation.

$$
\mathrm{V}_{\mathrm{i}, \mathrm{j}}=\mathrm{z}_{\mathrm{i}, \mathrm{j}}+\phi_{\mathrm{ij}}\left(\mathrm{z}_{\mathrm{i}, \mathrm{j}}-\mathrm{z}_{\mathrm{k}, \mathrm{j}}\right)
$$

Where $\mathrm{k} \varepsilon(1,2 \ldots \mathrm{N})$ and $\mathrm{j} \varepsilon(1,2 \ldots \mathrm{D})$ is a randomly produced number in the range $[-1,1]$ and

$v_{i, j}$ is the new value of the $j^{\text {th }}$ position. Then the fitness value is computed for every new generated population parameters of food sources. From the computed fitness value of the population, best population parameter is selected. The population parameter which has the highest fitness value by applying greed selection process. After selecting the best population parameter, the probability of the selecting parameter is computed using the equation,

$\mathrm{P}_{\mathrm{j}}=\frac{F^{v m}(j)}{\sum_{j=1}^{d} F^{v m}(j)}$

Where,

$P_{j}$ is the probability of the $\mathrm{j}^{\text {th }}$ parameter.

Onlooker bee phase: In the Onlooker bee phase after computing the probability of the selected parameter, the numbers of onlooker bees are estimated. Following, generate new solutions $\left(v_{i, j}\right)$ for the onlooker bees from the solutions $\left(\mathrm{z}_{\mathrm{i}, \mathrm{j}}\right)$ based on the probability value $\left(\mathrm{p}_{\mathrm{j}}\right)$ then the fitness function is calculated for the new solution. Subsequently apply the greedy selection process in order to select the best parameter.

Scout bee phase: In the Scout bee phase determine the abandoned parameters for the scout bees. If any abandoned parameter is present, then replace that with the new parameters discovered by scouts using the equation (4) and evaluate the fitness value. Then memorize the best parameters achieved so far. The above process is repeated until it reaches the number of iterations. Once it reaches, the best parameters are selected. Among the best parameters, some parameters contain same index values. This reputation of index values creates complexity in the future process. So, the repeated index values are taken on one occasion and the remaining repeated values are eliminated.

In Fig.3 shows the flowchart of implementation of Artificial Bee Colony algorithm for optimization for finding a vehicle optimal path. In the initialization phase, the control parameters are set, such as colony size, iteration number (bee travel time), working to onlooker bee rate.

In the next phase, the map is given as an input to the vehicle with the number of locations that are to be visited by the vehicle. Then path is obtained by using nearest neighbor method. Further when the working bees are initialized, the bee optimization loop is set. Then the random node is assigned for the bee to start, then by computing the probabilities given by equation (4) the bees will work and draw the next node to obtain the path and will memorize the best solution found so far using the greedy selection strategy.
Finally the bees become scout bees and the number of working bees is updated, that is the employed bee which is exhausted becomes the scout bee again. The optimization loop is terminated when the numbers of iterations are completed and the best result is obtained. The scout bees then again start to search for the new path.

\subsection{To Train the Vehicle Optimal Path by FFBNN}

For above process, the number of optimal path index values and these optimal paths are employed to determine the find the vehicle future location by FFBNN. The neural network is well trained in using these optimal paths index values are selected from the vehicle best parameter in order to predict future location. The neural network consists of two input units, $\mathrm{N}_{\mathrm{d}}$ hidden units and one output unit. The structure of the

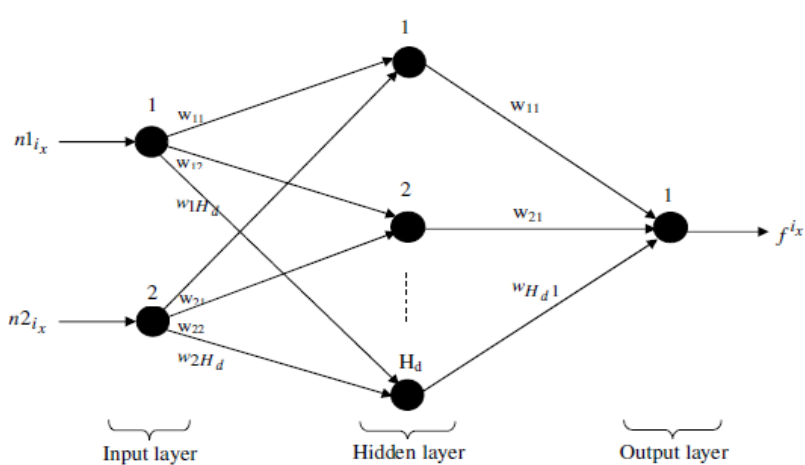

Fig 4.Diagram of the FFBNN

For all the neurons, allocate weights arbitrarily except for input neurons.

$\checkmark \quad$ In bias function and activation function of the neural network is described below.

$$
\begin{aligned}
& \mathrm{Y}_{\mathrm{ix}}=\beta+\sum_{h=1}^{H_{d}}\left(w_{h} n 1+w_{h} n 2\right) \\
& \alpha=\frac{1}{1+e^{-y_{i x}}}
\end{aligned}
$$

In bias function $\mathrm{n} 1, \mathrm{n} 2$ is the input node values of the index ix. The activation function for the output layer is given in equation (6)

Get the learning error.

$$
\gamma=\frac{1}{H_{D}} \sum_{h=0}^{H_{d}-1}\left(D_{h}-F_{h}\right)
$$

$\gamma$ is the FFBNN network output $D_{h}-F_{h}$ are desired and actual outputs and the error between the nodes are transmitted back to the hidden layer and this process is called the backward pass of the back propagation algorithm.

\section{Error Reduction}

Weights are assigned to the hidden layer and output layers neurons by randomly chosen weights. The input layer neurons have a constant weight.

- Determine the bias function and the activation function.

- To Compute $\mathrm{BP}_{\mathrm{e}}$ error for each node and update the weights as follows: 


$$
\mathrm{W}_{\mathrm{h}=} \mathrm{W}_{\mathrm{h}+} \Delta \mathrm{w}_{\mathrm{h}}
$$

$\Delta w h$ is obtained as,

$$
\Delta \mathrm{w}_{\mathrm{h}}=\delta . \text { Yix. B } \mathrm{p}_{\mathrm{e}}
$$

Where $\delta$ is the learning rate, which usually ranges from 0.2 to 0.5 and $\mathrm{Bp}_{\mathrm{e}}$ is the Back Propagation error.

- Then repeat the bias function and the activation function, the BP error calculation process is continued till the $\mathrm{Bp}$ error gets reduced ie. $\mathrm{Bp}_{\mathrm{e}}<0.1$

The error gets minimized to a minimum value the FFBNN is well trained in performing the moving vehicle location prediction and provides an appropriate fitness value for the respective input path values.

In order to obtain higher accuracy and efficient operation in vehicle future location prediction, the FFBNN parameters $\left(\mathrm{W}_{\mathrm{h}}, \beta\right)$ are optimized using $\mathrm{ABC}$.

\subsection{Optimization of FFBNN parameters by $\mathrm{ABC}$}

At present, to applying the $\mathrm{ABC}$ algorithm again for optimizing the parameters of FFBNN while training itself to get efficient prediction results. The FFBNN parameters $\left(\mathrm{W}_{\mathrm{h}}\right.$, $\beta$ ) are optimized using $A B C$. This optimization of FFBNN parameters using $\mathrm{ABC}$ gives higher vehicle future location prediction result and effective performance. In the test procedure, various vehicle optimal paths are given to well train FFBNN-ABC to check whether the test data will predict the vehicle's future location exactly or not. The same procedure is followed for all vehicles to predict the future location efficiently and more accurately.

\section{EXPERIMENTAL RESULT}

The proposed technique for prediction of moving vehicle location with FFBNN-ABC is implemented in the working platform of MATLAB (version 7.12). The proposed technique is the combination of FFBNN and ABC. In order to reduce the computation complexity and get higher performance, the proposed technique accurately finds the moving vehicle's location by finding their frequent paths. Here, all the vehicles frequent moving paths are collected and then optimal frequent paths of each vehicle are computed by Artificial Bee Colony technique and each vehicle frequent path is trained in the FFBNN and afterward in performance testing, the vehicles future location is predicted. Five vehicles frequently moving paths are composed at a certain time period, which is listed in the Table 2 .

Table 2: Sample frequent paths of five vehicles

\begin{tabular}{|c|c|c|c|c|c|}
\hline Vehicles & V1 & V2 & V3 & V4 & V5 \\
\hline $\begin{array}{c}\text { Frequent } \\
\text { Paths }\end{array}$ & $1-4$ & $1-8$ & $6-10$ & $6-10$ & $4-9$ \\
\cline { 2 - 6 } & $4-6$ & $3-9$ & $6-8$ & $8-7$ & $7-10$ \\
\hline
\end{tabular}

By using these frequent paths, the optimal frequent paths are computed and given to the FFBNN. The sample moving paths of vehicle $\mathrm{v} 1$ is show in the following Fig 5 .

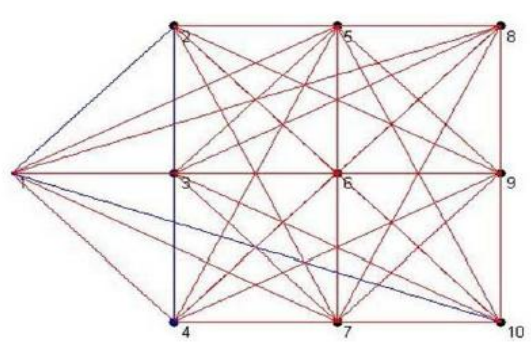

Fig 5.Sample Moving Vehicle A

At this time, to find all the vehicle's optimal frequent paths and these optimal paths of each vehicle are then trained in FFBNN. This well trained FFBNN is used in the testing process. The proposed technique is tested with five numbers of vehicles and the predicted results are given in the following Table 3.

Table 3: Vehicle Optimal Paths discovered by FFBNNABC

\begin{tabular}{|c|cc|cc|cc|cc|cc|}
\hline Vehicles & \multicolumn{10}{|c|}{ Optimal Frequent Paths } \\
\hline A & 10 & 9 & 4 & 10 & 4 & 2 & 5 & 6 & 3 & 2 \\
\hline B & 2 & 9 & 7 & 5 & 2 & 3 & 7 & 4 & 9 & 3 \\
\hline C & 1 & 8 & 1 & 5 & 3 & 6 & 2 & 9 & 10 & 8 \\
\hline D & 3 & 4 & 6 & 2 & 5 & 4 & 8 & 3 & 7 & 4 \\
\hline E & 10 & 1 & 3 & 9 & 8 & 6 & 7 & 10 & 4 & 6 \\
\hline
\end{tabular}

The proposed technique's performance is compared with the FFBNN-GA (Genetic Algorithm), FFBNN-PSO (Particle Swarm Optimization).The optimal paths are given in the following Table 4 and Table 5 is given to the training process and the trained GA and PSO (REF) is tested with moving vehicles. 
Table 4: Optimal Paths discovered by FFBNN-PSO

\begin{tabular}{|c|cc|cc|cc|cc|cc|}
\hline Vehicles & \multicolumn{10}{|c|}{ Optimal Frequent Paths } \\
\hline A & 9 & 4 & 4 & 6 & 1 & 2 & 3 & 2 & 5 & 10 \\
\hline B & 3 & 9 & 5 & 10 & 2 & 9 & 6 & 4 & 2 & 8 \\
\hline C & 4 & 5 & 2 & 1 & 5 & 10 & 10 & 7 & 4 & 2 \\
\hline D & 5 & 6 & 8 & 3 & 10 & 3 & 1 & 6 & 10 & 6 \\
\hline E & 7 & 2 & 7 & 9 & 6 & 8 & 8 & 5 & 2 & 4 \\
\hline
\end{tabular}

Table 5: Optimal Paths discovered by FFBNN-GA

\begin{tabular}{|c|cc|cc|cc|cc|cc|}
\hline $\begin{array}{c}\text { Vehicle } \\
\text { s }\end{array}$ & \multicolumn{10}{|c|}{ Optimal Frequent Paths } \\
\hline A & 10 & 8 & 1 & 8 & 1 & 6 & 3 & 2 & 5 & 10 \\
\hline B & 3 & 10 & 8 & 6 & 3 & 5 & 6 & 4 & 2 & 8 \\
\hline C & 4 & 2 & 2 & 3 & 6 & 2 & 10 & 8 & 4 & 6 \\
\hline D & 2 & 6 & 9 & 3 & 2 & 4 & 1 & 6 & 10 & 4 \\
\hline E & 7 & 5 & 7 & 4 & 5 & 7 & 8 & 5 & 8 & 4 \\
\hline
\end{tabular}

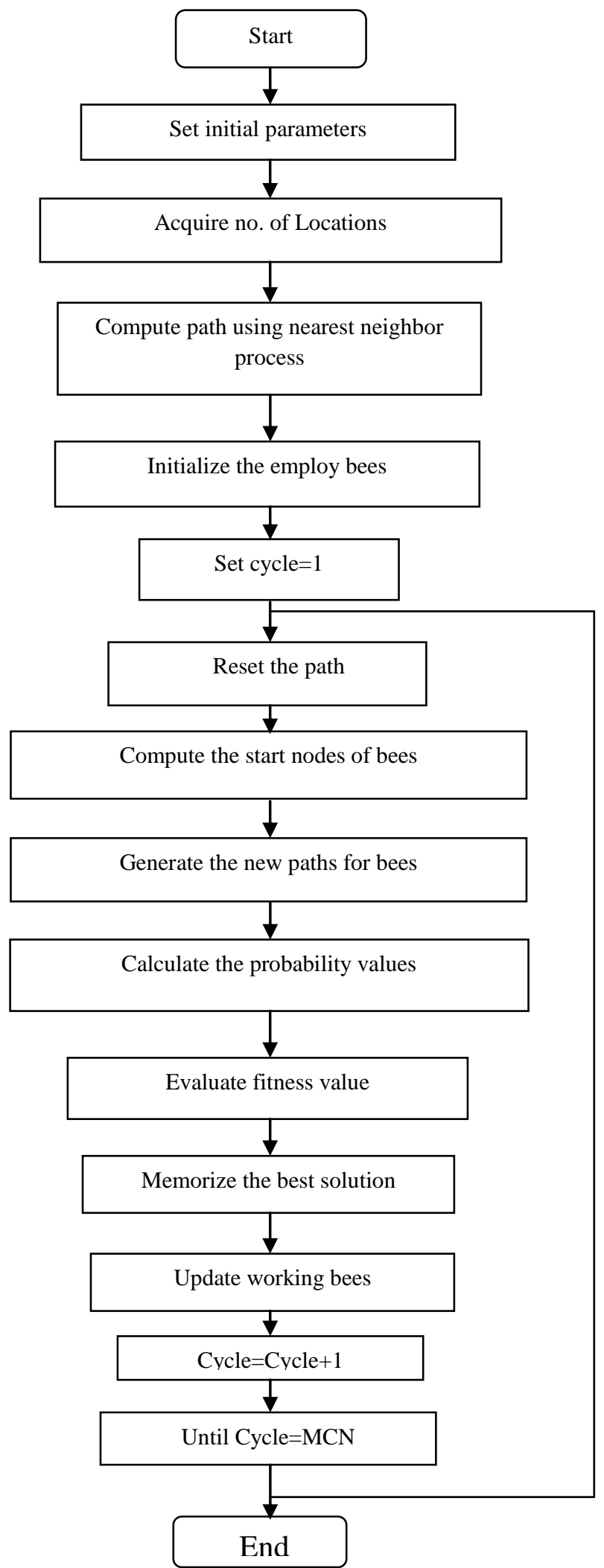

Fig 3. The flowchart of $\mathrm{ABC}$ algorithm for finding a vehicle optimal path 
In addition, the vehicle prediction accuracy measures of our proposed and existing systems are given in Table 6.The vehicle prediction accuracy is calculated by utilizing the formula,

$$
\text { Accuracy }=\frac{C^{\text {pat } h}}{N^{\text {pat } h}}
$$

Where, $\mathrm{C}^{\text {path }}$ is a correctly predicted path

$\mathrm{N}^{\text {path }}$ is a total number of optimal paths

Table 6: Performance of proposed moving vehicle location prediction techniques and other Optimization technique such as FFBNN-PSO, FFBNN-GA

\begin{tabular}{|c|c|c|c|}
\hline \multirow{2}{*}{ Vehicles } & \multicolumn{3}{|c|}{ Accuracy } \\
\cline { 2 - 4 } & $\begin{array}{c}\text { Proposed } \\
\text { FFBNN-ABC }\end{array}$ & FFBNN-PSO & FFBNN-GA \\
\hline V1 & 80 & 60 & 60 \\
\hline V2 & 100 & 100 & 80 \\
\hline V3 & 80 & 60 & 40 \\
\hline V4 & 100 & 80 & 60 \\
\hline V5 & 100 & 60 & 40 \\
\hline
\end{tabular}

The comparison result of both methods performance measures are given in the Fig 6.

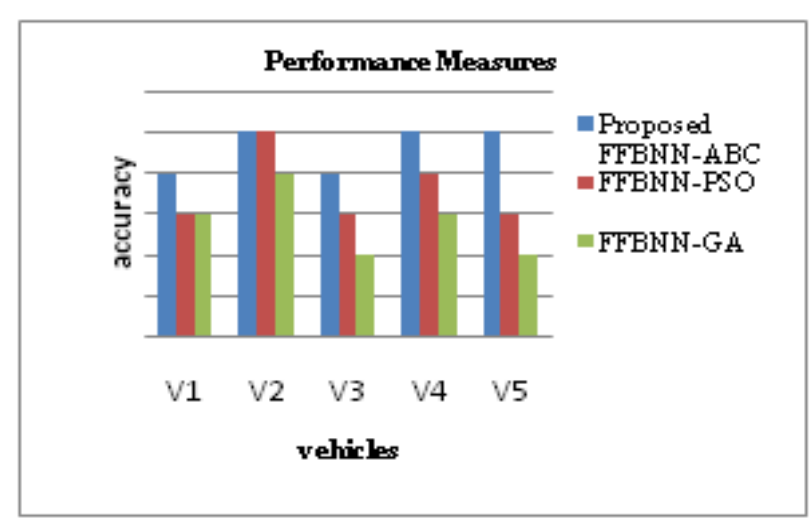

Fig 6: Prediction Accuracy for Proposed and Existing Methods Using Graph.

In Tables 6 accuracy measures are given. The accuracy of the proposed technique is $98 \%$.FFBNN-PSO; FFBNN-GA has $95 \%$ and $88 \%$ of accuracy respectively. When compared to the proposed FFBNN-ABC technique, FFBNN-PSO has 5\% lesser accuracy; FFBNN-GA has $12 \%$ lower accuracy. It represents the proposed technique predict the vehicle future location more accurately than the other techniques. In Fig 4, the accuracy of the proposed technique is compared with the other techniques. By considering the graph, the accuracy of the proposed technique is significantly higher than the FFBNN-GA, FFBNN-PSO. Here all five vehicles prediction accuracy results are higher than the previously used method. The comparison results shows that the proposed FFBNN$\mathrm{ABC}$ moving vehicle location prediction technique more precisely determine the moving vehicle future location than other technique.

\section{CONCLUSION}

In this paper, the proposed moving vehicle location prediction technique with aid of FFBNN and ABC. The proposed system was implemented and a huge set of test data were used to analyze the outcomes of the proposed system. Thus proposed system offers a significant tempo of accuracy. The proposed method more precisely predict the vehicle movement from the given test data by seeing the eminent rate of measurements. The comparison result shows that our proposed moving vehicle future prediction system based on FFBNN-ABC has given high accuracy than FFBNN- GA, FFBNN-PSO methods. Thus proposed algorithm has offered better performance in predicting the vehicle's future location with higher accuracy. In future, this work can also be extended to prediction of vehicle co-location.

\section{REFERENCES}

[1] Li, D. and S.Wang, "Concepts, Principles and Applications of Spatial Data Mining and Knowledge Discovery", Proceedings of the International Symposium on Spatio-Temporal Modeling, (STM'05), Beijing, China, pp: 1-13, 2005.

[2] Diansheng, G. and J. Mennis, "Spatial data mining and geographic knowledge discovery-An introduction" Comput. Environ.Urban, 2009.

[3] Buang, N., M.Z. Abdullahand and M.S. Zakaria, "Exploring spatial relationships for knowledge discovery in spatial data" Proceedings of the International Conference on Computer Engineering and Applications, (CEA' 11), IACSIT Press, Singapore, pp: 487-491, 2011.

[4] Brakatsoulas, S., D.Pfoser, R. Salas and C.Wenk," On map-matching vehicle tracking data" Proceedings of the 31st International Conference on Very Large Data Bases, Oct. 04-06, ACM Press, pp: 853-864, 2005.

[5] Liu, H. and M.Schneider, "Tracking continuous topological changes of complex moving regions" Proceedings of the ACM Symposium on Applied Computing, Mar. 21-24, ACM Press, New York, pp: 833-838, 2011.

[6] Shaw, A.A. and N.P. Gopalan "Frequent pattern mining of trajectory coordinates using apriori algorithm" Int. J. Comput. Appl., 22: 1-7, 2011.

[7] Ivana Nizetic and Kresimir Fertalj, "Automation of the Moving Objects Movement Prediction Process Independent of the Application Area" Computer. Sci. Inf. Vol. 7, Issue 4, 2010

[8] D.Malerba11, "Mining Spatial Data: Opportunities and Challenges of a Relational Approach" IASC 07, 2007.

[9] Ajaya Kumar Akasapu, Lokesh Kumar Sharma, G.Ramakrishna, "Efficient Trajectory Pattern Mining for both sparse and Dense Dataset" International Journal of Computer Applications Volume 9, 2010, pp.5.

[10] Ashita S. Bhagade, Parag. V. Puranik, "Artificial Bee Colony (ABC) Algorithm for Vehicle Routing Optimization Problem", International Journal of Soft Computing and Engineering (IJSCE) ISSN: 2231-2307, Volume-2, Issue-2, May 2012.

[11] Dervis Karaboga, Celal Ozturk, "A novel clustering approach: Artificial Bee Colony (ABC) algorithm" Applied Soft Computing 11 (2011) 652-657 
[12] K.Balachandran, Dr. R. Anitha, “An Efficient Optimization Based Lung Cancer Pre-Diagnosis System with Aid of Feed Forward Back Propagation Neural Network (FFBNN)" Journal of Theoretical and Applied Information Technology 20th October 2013.Vol. 56 No.2

[13] Milan TUBA, Nebojsa BACANIN, Nadezda STANAREVIC, "Adjusted artificial bee colony (ABC) algorithm for engineering Problems" WSEAS Transactions on Computers, Issue 4, Volume 11, April 2012.

[14] Bahriye Akay, "A study on particle swarm optimization and artificial bee colony algorithms for multilevel thresholding" Applied Soft Computing (2013) 30663091.

[15] Ginnu George, Kumudha Raimond, "A Survey on OptimizationAlgorithms for Optimizing the Numerical Functions" Internationa Journal of Computer Applications (0975 - 8887) Volume 61- No.6, January 2013.
[16] W.Y. Szeto, Yongzhong Wu, Sin C. Ho, “An artificial bee colony algorithm for the capacitated vehicle routing problem" European Journal of Operational Research 215 (2011) 126 - 135.

[17] E.Baby Anitha, Dr.K.Duraiswamy, "Prediction of Vehicle Movement Using Spatial Mining: A Recent Survey" International Journal of Advanced Research in Technology, Vol. 2 Issues 4, 2012.

[18] E.Baby Anitha, Dr.K.Duraiswamy, "A heuristic moving vehicle location prediction technique via optimal paths selection with aid of genetic algorithm and feed forward back propagation neural network" Journal of Computer Science Vol 8 (12), 2012, pp.2008-2016.

[19] E. Baby Anitha, Dr.K.Duraiswamy, “A new hybrid approach for prediction of moving vehicle location using particle swarm optimization and neural network" Journal of Theoretical and Applied Information Technology, Vol 59, No.3, pp 791-800, 2014. 